\title{
Poly(ethylene imine) impurities induce n-doping reaction in organic (semi)conductors
}

\author{
Simone Fabiano, Slawomir Braun, Xianjie Liu, Eric Weverberghs, Pascal Gerbaux, Mats \\ Fahlman, Magnus Berggren and Xavier Crispin
}

\section{Linköping University Post Print}

\section{Tweet}

N.B.: When citing this work, cite the original article.

Original Publication:

Simone Fabiano, Slawomir Braun, Xianjie Liu, Eric Weverberghs, Pascal Gerbaux, Mats Fahlman, Magnus Berggren and Xavier Crispin, Poly(ethylene imine) impurities induce ndoping reaction in organic (semi)conductors, 2014, Advanced Materials, (26), 34, 6000-6006. http://dx.doi.org/10.1002/adma.201401986

Copyright: Wiley-VCH Verlag http://www.wiley-vch.de/publish/en/

Postprint available at: Linköping University Electronic Press http://urn.kb.se/resolve?urn=urn:nbn:se:liu:diva-111452 


\section{WILEY-VCH}

DOI: $10.1002 /($ please add manuscript number $))$

Article type: Communication

Poly(ethylene imine) impurities induce n-doping reaction in organic (semi)conductors

Simone Fabiano, Slawomir Braun, Xianjie Liu, Eric Weverberghs, Pascal Gerbaux, Mats Fahlman, Magnus Berggren and Xavier Crispin*

Dr. S. Fabiano, Prof. M. Berggren, Prof. X. Crispin

Laboratory of Organic Electronics, Department of Science and Technology, Linköping

University, SE-601 74 Norrköping, Sweden

E-mail: xavier.crispin@liu.se

Dr. S. Braun, Dr. X. Liu, Prof. M. Fahlman

Department of Physics, Chemistry and Biology, Linköping University, SE-581 83 Linköping, Sweden

Dr. E. Weverberghs, Prof. P. Gerbaux

Organic Synthesis and Mass Spectrometry Laboratory, University of Mons-UMONS, B-7000

Mons, Belgium

Keywords: amines, electron transfer, doping, charge injection, organic electronics 


\section{WILEY-VCH}

Organic semiconductors have been attracting considerable research interest as building blocks in emerging optoelectronic devices such as light-emitting diodes (OLEDs), ${ }^{[1]}$ polymer solar cells (PSCs), ${ }^{[2]}$ and field-effect transistors (OFETs). ${ }^{[3]}$ The operation of such organicbased devices involves the injection of charges into the organic semiconductor, which typically are sourced from a metallic electrode. This charge injection process plays a dominant role in determining the device efficiency. ${ }^{[4]}$ Thus, a thorough understanding of the material parameters that limits the charge injection characteristics (i.e. morphology, electrical properties, and energetics of the metal/organic interfaces) is crucial to optimize the device performance. In general, the energetics at the contact plays an important role in determining the charge injection efficiency. Charges are injected into the semiconductor if energy barriers are overcome. Typically, easy hole [electron] injection into the organic semiconductor is achieved by choosing an electrode with a work function that matches the highest occupied molecular orbital (HOMO) [lowest unoccupied molecular orbital (LUMO)] of the organic semiconductor. ${ }^{[5]}$ The charge injection efficiency is shown to increase dramatically as the barrier height at the interface decreases. ${ }^{[6]}$ Therefore, minimizing the energy barrier at the metal/organic semiconductor interface help to make the contact ohmic, which enables high injection currents already at low voltages. ${ }^{[7]}$ This is a well-known rule of design for organic semiconductor devices, which leads to the fact that low (high) work function metals are used for electron (hole) injection. Several strategies have been explored to improve the charge injection efficiency, such as interfacial dipole formation, ${ }^{[8]}$ variation of the molecular orientation at the semiconductor/electrode interface, ${ }^{[9]}$ presence of mobile ions near the contacts, ${ }^{[10]}$ doping of the organic semiconductor bulk ${ }^{[11]}$ or at the interface with the electrode. ${ }^{[12]}$ In addition, molecular layers expressing an intrinsic electric dipole moment (e.g. self-assembled monolayers) can be used to lower or raise the work function of the electrodes, thus affecting the magnitude of the charge injection. ${ }^{[13]}$ 


\section{WILEY-VCH}

Low work-function electrodes are typically needed to inject electrons, at low voltages, into the LUMO level of the organic semiconductor. However, this implies the use of thin metallic interlayers as for instance calcium ${ }^{[14]}$ or lithium fluoride, ${ }^{[15]}$ which are chemically reactive and easily oxidize in the presence of ambient oxygen and water. Recently, insulating polymers containing simple aliphatic amine groups such as the highly branched polyethylenimine (PEI, scheme 1a) have been shown to be universal surface modifiers that allow the fabrication of air-stable low-work function electrodes for efficient electron injection. ${ }^{[16]}$ This novel approach enables the mass production of electron-injecting electrodes, which are stable in ambient condition, from processes that are compatible with large-area manufacturing techniques, as ultrathin layers of such large band-gap insulating polymers can be simply printed onto the conductor surface. Enhancement in efficiency of various optoelectronic devices, which includes ultra-thin PEI interfacial layers, has been demonstrated for several devices. The improvement in efficiency is explained in terms of a better energy level alignment between the metal electrode and the organic semiconductor. The intrinsic molecular dipole moments, associated with the neutral amine groups contained in the insulating polymer, is expected to reduce the barrier for electron injection. ${ }^{[16]}$

Here, we show that the vapor from the PEI sample contains N-based impurity molecules (hereafter referred to as "PEI-vapor") with a strong reducing character and that this is the prime mechanism behind making the electron injection efficient. A chemical reaction occurs at the PEI-vapor/organic semiconductor or at the PEI-vapor/electrode interfaces. In some cases we actually observe that the volatile molecular reducing agent, from the PEI sample, migrates throughout the entire bulk of the organic material. Hence, both the energetics at the interface and the electrical conductivity of the organic (semi)conductor bulk are changed. We believe that our findings provide understanding of the origin behind the efficiency improvement measured in organic (opto)electronic devices including PEI as an interfacial layer. 


\section{WILEY-VCH}

Note that chemical doping of organic semiconductors has been shown to be an effective method to improve device performance by increasing the conductivity and enhancing charge injection from electrodes. ${ }^{[17]}$ Due to the high density of electron-donating amine groups, PEI has been successfully used to n-dope carbon nanotubes and graphene. ${ }^{[18]}$

Here, we investigate the effect caused by the vapor of PEI samples (Sigma-Aldrich CAS Number 25987-06-8 and 9002-98-6, same as used in previous studies ${ }^{[16]}$ ) on the electronic properties of three key materials normally included in organic (opto)electronic devices: the p-type conductor poly(3,4-ethylenedioxythiophene)-poly(styrene sulfonate) (PEDOT-PSS, scheme 1b) commonly used as a transparent polymer electrode, ${ }^{[19]}$ the $n$-type semiconductor [6,6]-phenyl-C61-butyric acid methyl ester (PCBM, scheme 1c) often used in PSCs, ${ }^{[20]}$ and poly\{[N,N'-bis(2-octyldodecyl)-naphthalene-1,4,5,8-bis(dicarboximide)-2,6diyl]-alt-5,5'-(2,2'-bithiophene) $\} \quad$ (P(NDI2OD-T2), scheme 1d) used in n-type organic OFETs. ${ }^{[21]}$ Both PCBM and P(NDI2OD-T2) have several compelling properties including high electron mobility and stability in air, which place them among the most comprehensively studied systems for OFETs ${ }^{[22]}$ and PSCs. ${ }^{[23]}$

We notice that when PEDOT-PSS is exposed to the vapor of the PEI sample, it turns dark blue (Figure 1a). This suggests that PEDOT reduces to its neutral state. Figure 1b shows the optical absorption spectra of a PEDOT-PSS film before (red) and after (blue) exposure to the PEI vapor. Before exposure to the vapor of the PEI sample, the absorption spectrum of the pristine PEDOT is composed of a broad absorption band that extend far into the near infrared region. This band is associated with the free charge carriers and the mid-gap states (polaron or bipolaron states) of the conducting polymer. ${ }^{[24]}$ However, after exposure to the vapor of the PEI sample, the PEDOT spectrum shows a pronounced optical absorption feature at around $600 \mathrm{~nm}$, which originates from the neutral state (HOMO-LUMO transition) and the bipolaronic absorption bands in the near infrared region are suppressed. ${ }^{[25]}$ Also, the appearance of a new absorption band at around $900 \mathrm{~nm}$ is also found, which is ascribed to the 


\section{WILEY-VCH}

polaron-induced absorption of PEDOT. These optical features are qualitatively consistent with previous reports on dedoping of PEDOT ${ }^{[19]}$ Note that a similar effect is also observed when PEDOT-PSS thin films are brought in direct contact with the PEI sample and is associated with an electron transfer from the amine groups of PEI to the oxidized PEDOT chains. ${ }^{[26]}$ Tertiary amines such as triethylamine do not show the same reducing character, ${ }^{[26]}$ most likely due to their weak donor character when compared to primary and secondary amines. $^{[27]}$

Further insight into the mechanism of dedoping of PEDOT caused by the exposure of the vapor of the PEI sample is here provided by XPS studies. Indeed, a strong N1s signal appears after exposure to the PEI vapor (Figure 2a), which indicates that the volatile molecules react with the PEDOT phase and that the reactant contains nitrogen atoms. The peak located at around $402 \mathrm{eV}$ supports the presence of positively charged amine groups (e.g. $\left.-\mathrm{NR}_{3}{ }^{+}\right),{ }^{[28]}$ corroborating the proposed $\mathrm{n}$-doping mechanism. To further investigate the detail of this interaction between the N-containing molecules and PEDOT, we turn to analyze the changes in the $\mathrm{S} 2 p$ core-level signal. The sulfur atom is represented by a doublet due to spinorbit coupling. The S2 $p$ XPS spectrum shows two major contributions, one from PEDOT at low binding energy (167-163 eV) and one from PSS at high binding energy (171-167 eV). The relatively higher binding energy of the sulfur atom in PSS is due to the fact that sulfur has a deficit in electron density due to the presence of three electronegative oxygen atoms in the sulfonate group. ${ }^{[29]}$ The photoelectron spectra of the PEDOT-PSS film before and after exposure to the PEI sample are given in Figure $2 b$. Deconvolution performed on the PEDOT component of the S2p signal of the pristine sample reveals strong asymmetry of the doublet (tail stretching towards higher binding energy), which is ascribed to the presence of positive charges [(bi)polaron defects] delocalized over PEDOT chains thus altering the electronic environment in the vicinity of the sulfur atom, which in turn modifies the $\mathrm{S} 2 p$ core level signal. ${ }^{[29]}$ This asymmetric tail is no longer observed upon exposure of the PEDOT film 


\section{WILEY-VCH}

sample to the vapor of the PEI sample, which confirms the drastic decrease in the oxidation level, i.e. an increase of neutral PEDOT segments. After the electron transfer from the Nbased molecular vapor $\left(\mathrm{RN}_{\mathrm{m}}\right)$ to the positively charged PEDOT chains, some PEDOT segment becomes neutral and the $\mathrm{N}$-based molecule becomes cationic and is stabilized by the PSS anions to form the equivalent of a salt PSS- $\mathrm{RN}_{\mathrm{m}}{ }^{+}$complex. Deconvolution of the PSS contribution of the spectra indicates a change in the chemical surrounding of the sulfur that comes from the sulfonate group of PSS. The XPS survey scan indicates also an increase of the $\mathrm{Na}$ content that occurs upon treatment, which can be rationalized by the $\mathrm{PSS}^{-}-\mathrm{Na}^{+}$conversion to PSS- $\mathrm{RN}_{\mathrm{m}}^{+}$and an associated release of $\mathrm{Na}^{+}$that then migrates to the surface and contributes to the Na1s signal (see Supporting Information, Figure S1).

Hence, the proposed reaction between PEDOT-PSS and the vapor of the PEI is a redox reaction where some low-molecular component of the PEI sample functions as a reducing agent by donating electrons to the $\mathrm{PEDOT}^{+}$, thus producing $\mathrm{PEDOT}^{0}$ and $\mathrm{PSS}_{\mathrm{RN}}{ }_{\mathrm{m}}^{+}$ complex.

The electrical properties of the PEDOT-PSS film were investigated before and after exposure to the vapor of the PEI sample by using standard four-point-probe as well as twoprobe current-voltage measurements (Figure 3a). The PEDOT-PSS film with a thickness of $d$ $=100 \mathrm{~nm}$ was spin-coated from a water-based ink (high-conductivity grade PH1000) onto glass substrate with pre-patterned gold contacts (see Experimental Section for further details). The films were then exposed to the vapor of the PEI sample inside a nitrogen-filled glovebox. Figure 3a shows the electrical conductivity of the PEDOT-PSS film as a function of the exposure time. Before exposure, the pristine PEDOT-PSS film shows an electrical conductivity as high as $4.0 \mathrm{~S} \mathrm{~cm}^{-1}$. However, the conductivity drops by more than one order of magnitude immediately after exposure to PEI vapor and reaches finally a value as low as $5.4 \times 10^{-4} \mathrm{~S} \mathrm{~cm}^{-1}$ after $30 \mathrm{~h}$ exposure at room temperature. Similar changes in electrical conductivity were observed for dedoped PEDOT upon reaction with strong reducing reagents 


\section{WILEY-VCH}

such as hydrazine. ${ }^{[30]}$ This observation is in agreement with the color change observed by optical measurements and clearly confirms successful dedoping of the PEDOT in the films upon exposure to the vapor of the PEI sample.

The observed decrease of PEDOT-PSS film conductivity is rationalized by the suppression of polaronic states upon charge donation from a low-molecular component of the PEI sample. This is confirmed by the changes in UPS spectra, shown in Figure $3 b$, where the density of states close to Fermi level is dramatically reduced indicating lack of free charge carriers in the film. Furthermore, as revealed by the secondary electron cutoff (SECO) spectra, the work function of the film decreases from $5.0 \mathrm{eV}$ to $3.7 \mathrm{eV}$ (Figure 3b). This will have a profound impact on energy level alignment at interfaces with other layers in an electronic device. ${ }^{[7]}$ We also tested the reversibility of this reduction process by re-measuring the conductivity as the PEDOT-PSS films are exposed to air. Indeed, due to its strong electrondonor character, neutral PEDOT undergoes oxygen-doping once exposed to ambient atmosphere. ${ }^{[26]}$ This results in a slight increase of the electrical conductivity (filled circle in Figure 3a) when the PEI-vapor-dedoped PEDOT-PSS film is removed from the nitrogenfilled glovebox to the ambience. However, the recovery is not complete and is likely due to the basic chemical character of the PEI vapor, which reacts with the sulfonic acid groups of PSS, thus preventing it to re-dope PEDOT.

Next, we investigate if the PEI vapor can also reduce neutral organic semiconductors. Compared to the conducting polymer PEDOT-PSS, organic semiconductors such as PCBM and P(NDI2OD-T2) are pristinely undoped. Indeed, similarly to other organic semiconductors, both PCBM and P(NDI2OD-T2) are unintentionally doped semiconductors with a very low conductivity in the undoped pristine state (ca. $\left.5 \times 10^{-8}-1 \times 10^{-7} \mathrm{~S} \mathrm{~cm}^{-1}\right)$. However, after exposure to the vapor of the PEI sample, the electrical conductivity of the semiconductors increases considerably and saturates rapidly to an average value of $5 \times 10^{-4} \mathrm{~S} \mathrm{~cm}^{-1}$ for PCBM (within 10 hours) and $8 \times 10^{-5} \mathrm{~S} \mathrm{~cm}^{-1}$ for $\mathrm{P}(\mathrm{NDI} 2 \mathrm{OD}-\mathrm{T} 2)$ (within 1 hour), being almost three to four 


\section{WILEY-VCH}

orders of magnitude higher that those of the undoped films (Figure 4). These values approach those reported for PCBM and P(NDI2OD-T2) films doped with benzoimidazolederivatives ${ }^{[31]}$ or $\left[\mathrm{RhCp}_{2}\right]_{2},{ }^{[32]}$ indicating the strong chemical doping ability of the volatile component of the PEI sample. Note that the rapid saturation of the electrical conductivity in PEI vapor-treated semiconductor films suggests that the n-doping effect is restricted only to the surface of the semiconducting layer. Indeed, unlike PEDOT-PSS where the doping process appears to be diffusion-limited due to the hydrophilicity of the PSS bulk, the hydrophobic character of PCBM and P(NDI2OD-T2) more likely prevents low molecular weight amines from penetrating the semiconducting bulk layer. This is corroborated by the optical measurements, showing no significant differences in the absorbance spectra of the pristine and treated films (see Figure S2). In addition, the XPS spectra further support this hypothesis as the nitrogen signal (taken at the same X-ray intensity and normalized by the number of scans) shows much smaller nitrogen content in the case of treated PCBM as compared to treated PEDOT-PSS films (see Figure S3). This suggests that the doping depth is smaller than the inelastic mean free path $\left(\sim 28 \AA^{[33]}\right)$. Note also that the high-conductivity state of the PCBM and P(NDI2OD-T2) films, after treatment, persists when the samples are kept under high vacuum $\left(\sim 10^{-7}\right.$ mbar, filled circles in Figure 4), indicating that the surface reaction with the PEI vapor is irreversible.

It is well established that doping can affect the physics of charge injection since it modifies the energy barrier at the contact. ${ }^{[5]}$ This means that charge injection in organic semiconductors depends directly on the charge density. In fact, the injection efficiency increases dramatically with doping and the contact becomes ohmic at high density of charges. Since doping can simultaneously improve the charge transport properties and enable the control of the barrier energetics for charge transfer at the organic/electrode and organic/organic interfaces, the incorporation into organic devices of amine-based layers such 


\section{WILEY-VCH}

as amino-functionalized polyfluorenes (e.g. $\mathrm{PFN}^{[34]}$ ) or fullerenes (e.g. $\mathrm{PC}_{71} \mathrm{BM}-\mathrm{N}^{[35]}$ ) can facilitate the possibility of ohmic contacts for electrons.

In order to identify the nature of the impurities of the PEI samples, we performed mass spectrometry (MS) analysis on the volatile molecules spontaneously emitted from the PEI samples. Here we utilize Electron Ionization (EI) in which the sample is charged by highly energetic electrons. The lowest molecular weight ions are first emitted and then analyzed. The procedure relies on the direct introduction (DI) of the non volatile PEI condensed phase into the EI ion source. Sampling of low-molecular weight molecules, which are evaporated, is then achieved at room temperature, i.e. without external heating, from the condensed phase under vacuum, typically at $10^{-5}$ mbar. As depicted in Figure 5a, numerous of ions are observed upon DI-EI-MS analysis of the low mass PEI, with a significant contribution of ionized PEI oligomers $(m / z$ 159-202-245-288...). These ions are readily identified as short ionized PEI oligomers based on the typical $43 \mathrm{u}$ residual mass. Obviously, the presence of these ions is not unexpected given the low $\mathrm{M}_{\mathrm{n}}$ (600 Da, as determined by Gel permeation Chromatography) of the PEI sample and the low ion source pressure. Several other ions also produce intense signals in the low mass range of the mass spectrum, such as at $m / z$ equals 44,56 and 87. These compounds are a priori not related to the direct ionization of volatile PEI short oligomers. High mass PEI $\left(\mathrm{M}_{\mathrm{n}}=10.000 \mathrm{Da}\right)$ was then subjected to the DI-EI-MS analysis and the spectrum presented in Figure 5b was recorded (see also Table S1, Supporting Information). Note that low and high mass PEI samples show the same n-doping effect of the organic (semi)conductors. Heavier PEI oligomer ions are no longer detected in the mass spectrum and interestingly the ions at $\mathrm{m} / \mathrm{z} 44,56$ and 99 are still present whereas additional ions are now clearly observed. Based on accurate mass measurements, $\left[\mathrm{C}_{\mathrm{n}} \mathrm{H}_{\mathrm{n}} \mathrm{N}_{\mathrm{p}}\right]^{+}$cations are identified with, for instance, $m / z$ 44, 56 and 73 being respectively $\mathrm{C}_{2} \mathrm{H}_{6} \mathrm{~N}^{+}, \mathrm{C}_{3} \mathrm{H}_{6} \mathrm{~N}^{+}$and $\mathrm{C}_{3} \mathrm{H}_{9} \mathrm{~N}_{2}{ }^{+}$. Based on the comparison with reference spectra from the NIST database, ${ }^{[36]}$ the recorded data are readily explained considering the presence of small ethyleneimine dimers or trimers, such as 


\section{WILEY-VCH}

$\mathrm{NH}_{2} \mathrm{CH}_{2} \mathrm{CH}_{2} \mathrm{NHCH}_{2} \mathrm{CH}_{2} \mathrm{NH}_{2}$, for instance. Indeed, $m / z, 44$ and 73 represent key signals in the

EI-MS mass spectrum of $\mathrm{NH}_{2} \mathrm{CH}_{2} \mathrm{CH}_{2} \mathrm{NHCH}_{2} \mathrm{CH}_{2} \mathrm{NH}_{2}$ and $\mathrm{NH}_{2} \mathrm{CH}_{2} \mathrm{CH}_{2}\left(\mathrm{NHCH}_{2} \mathrm{CH}_{2}\right)_{2} \mathrm{NH}_{2}$, whereas $m / z \quad 99$ and 116 signals are becoming prominent for branched tris(aminoethyl)amine. ${ }^{[36]}$ In the direct continuity of the analysis, the presence in the gas phase of heavier ethylene imine oligomers, either linear or branched, is confirmed by the occurrence of two distinct ion series; (i) $m / z \quad 56-99-142-185-228$ and (ii) $m / z \quad 73-116-159-202$. We validated this hypothesis by exposing the PEDOT-PSS films to the vapor of pure diethylenetriamine (Figure S5). Remarkably, the electrical conductivity drops by more than 5 orders of magnitude and the PEDOT-PSS films turn dark blue, confirming the strong reducing character of the diethylenetriamine vapor.

In conclusion, we demonstrated the presence of volatile impurities in polyethyleneimine (PEI), identified as ethyleneimine dimers and trimers. These N-based molecules show a strong reducing character, as demonstrated by the change in electrical conductivity for organic (semi)conductors that are exposed to the PEI sample vapor. Here, we have proven that the reduction of PEDOT (i.e. dedoping) extends throughout the entire bulk of the organic electrode material, while for PCBM and P(NDI2OD-T2) the n-doping reaction is confined only to the surface.

The presence of interfacial dipoles at the PEI/electrode interface has been cited many times as the reason for the enhanced device performances. However, the real mechanism responsible for such a performance appears to be more complex. Firstly, our results clearly suggest that the small volatile ethyleneimine dimer or trimer impurities contained in the PEI can lead to an efficient electron transfer at the interface with inorganic, metallic and organic electrodes. This leads to the creation of an interface dipole that decreases the effective electrode work function. Secondly, the interfacial n-doping effect observed in the organic semiconductors by the PEI impurities can explain the performance enhancement in (opto)electronic devices upon inclusion of the ultrathin PEI layer. 


\section{WILEY-VCH}

\section{Experimental Section}

Sample Preparation: High-conductivity grade PEDOT-PSS (Clevios PH1000, H.C. Starck $\mathrm{GmbH}$ ) was filtered using a $0.45 \mu \mathrm{m}$ hydrophilic syringe filter. The filtrate was spin-coated onto glass substrates at a speed of $1500 \mathrm{rpm}$ for $60 \mathrm{~s}$ and then dried at $140{ }^{\circ} \mathrm{C}$ for $10 \mathrm{~min}$ in an oven. A thickness of $100 \mathrm{~nm}$ was measured by using a DekTak 3ST profilometer (Veeco, NY). Thin films of PCBM (Solenne BV) and P(NDI2OD-T2) (ActivInk N2200, supplied by Polyera Corporation) were fabricated by spin coating from chlorobenzene $\left(8-10 \mathrm{mg} \mathrm{mL} \mathrm{m}^{-1}\right)$ onto glass substrates inside a $\mathrm{N}_{2}$-filled glovebox. The semiconductor solution was filtered with a $0.2 \mu \mathrm{m}$ PTFE syringe filter, spin cast at $1000 \mathrm{rpm}$ for $90 \mathrm{~s}$ and dried on a hot plate at $110{ }^{\circ} \mathrm{C}$ for $1 \mathrm{~h}$. This results in a layer thickness of about 30-50 nm for PCBM and 60-100 nm for P(NDI2OD-T2). For conductivity measurements, 30-nm-thick Au electrodes with a 5-nmthick Ti adhesion layer (1 mm channel length and $10 \mathrm{~mm}$ channel width) were fabricated on glass substrates prior to active layer deposition. All substrates were cleaned sequentially in deionized water, acetone and isopropanol, then treated with a UV-ozone plasma for $10 \mathrm{~min}$ after baking at $140{ }^{\circ} \mathrm{C}$ in oven. For PCBM and P(NDI2OD-T2), glass substrates were modified with hexamethyldisilazane (HMDS). Doping was conducted inside a $\mathrm{N}_{2}$-filled glovebox by exposing the samples to the vapor of a highly branched PEI of different molecular weights $\left(\mathrm{M}_{\mathrm{n}} / \mathrm{M}_{\mathrm{w}} \sim 600 / 800\right.$ and $\mathrm{M}_{\mathrm{n}} / \mathrm{M}_{\mathrm{w}} \sim 10.000 / 25.000$, purchased from SigmaAldrich). Diethylenetriamine 99\% was purchased from Sigma-Aldrich.

Characterization: Optical absorption measurements were conducted at room temperature under ambient atmosphere using an UV/Vis/NIR spectrophotometer (PerkinElmer Lambda 900). Current-voltage characteristics were measured using a semiconductor parameter analyzer (Keithley 4200-SCS).

Photoemission experiments were carried out using a Scienta ESCA 200 spectrometer under ultrahigh vacuum conditions at a base pressure of $1 \times 10^{-10}$ mbar. The XPS and UPS measurements have been done respectively with a monochromatic $\mathrm{Al} \mathrm{K} \alpha \mathrm{X}$-ray source, 


\section{WILEY-VCH}

providing photons with energy of $1,486.6 \mathrm{eV}$ and un-monochromatized source of HeI radiation $(\mathrm{h} v=21.2 \mathrm{eV})$. The work function values are derived from the on-set of the secondary electron cut-off. The XPS spectra are normalized to the $\mathrm{C} 1 s$ peak. Deconvolution of the $\mathrm{S} 2 p$ spectra was carried out on the basis of a priori knowledge of the chemical composition, and on the results reported in the literature. ${ }^{[29]}$ During the deconvolution performed on the $\mathrm{S} 2 p$ spectra, the energy difference between the $\mathrm{S} 2 p_{1 / 2}$ and $\mathrm{S} 2 p_{3 / 2}$ spin-orbit components was kept at $1.2 \mathrm{eV}$, and the intensity ratio fixed at 1:2.

DIP-EI-MS spectra were recorded on a large-scale tandem mass spectrometer (AutoSpec 6F, Waters) combining six sectors of E1B1c1E2c2E3B2c3E4 configuration (E stands for electric sector, B for magnetic sector and $\mathrm{c}$ for collision cell). General conditions were $8 \mathrm{kV}$ accelerating voltage, $70 \mathrm{eV}$ ionizing electron energy and $200{ }^{\circ} \mathrm{C}$ ion source temperature. The PEI samples (1 mg), introduced in small Pyrex capillaries, were directly introduced into the ion source without any external heating. The ions were obtained under electron ionization in positive ion mode (200 $\mu \mathrm{A}$ trap current). The full scan mass spectra were recorded by scanning the field of the first magnetic sector and collecting the ions with an off-axis photomultiplier detector. $^{[37]}$

\section{Supporting Information}

Supporting Information is available from the Wiley Online Library or from the author.

\section{Acknowledgements}

This work was partially supported by the Advanced Functional Materials Center at Linköping University and the Önnesjö Foundation. Authors wish to thank also the Knut and Alice Wallenberg Foundation (Power Paper project, scholars), VINNOVA, and STEM, the Swedish Energy Agency for financial support. PG strongly acknowledge the FRS-FNRS for financial support for the acquisition of the AutoSpec $6 \mathrm{~F}$ and for continuing support.

Received: ((will be filled in by the editorial staff))

Revised: ((will be filled in by the editorial staff)) Published online: ((will be filled in by the editorial staff)) 


\section{WILEY-VCH}

[1] A. C. Grimsdale, K. L. Chan, R. E. Martin, P. G. Jokisz, A. B. Holmes, Chem. Rev. 2009, 109, 897.

[2] A. Marrocchi, D. Lanari, A. Facchetti, L. Vaccaro, Energy Environ. Sci. 2012, 5, 8457; A. Facchetti, Mater. Today 2013, 16, 123.

[3] A. Facchetti, Chem. Mater. 2011, 23, 733; A. C. Arias, J. D. MacKenzie, I. McCulloch, J. Rivnay, A. Salleo, Chem. Rev. 2010, 110, 3.

[4] J. C. Scott, J. Vac. Sci. Technol. A 2003, 21, 521.

[5] Y. L. Shen, A. R. Hosseini, M. H. Wong, G. G. Malliaras, ChemPhysChem 2004, 5, 16.

[6] T. Matsushima, Y. Kinoshita, H. Murata, Appl. Phys. Lett. 2007, 91, 3.

[7] S. Braun, W. R. Salaneck, M. Fahlman, Adv. Mater. 2009, 21, 1450.

[8] S. Duhm, G. Heimel, I. Salzmann, H. Glowatzki, R. L. Johnson, A. Vollmer, J. P. Rabe, N. Koch, Nature Mater. 2008, 7, 326.

[9] S. Fabiano, H. Yoshida, Z. H. Chen, A. Facchetti, M. A. Loi, ACS Appl. Mater. Interfaces 2013, 5, 4417.

[10] S. Fabiano, S. Braun, M. Fahlman, X. Crispin, M. Berggren, Adv. Funct. Mater. 2014, 24,695 .

[11] B. H. Hamadani, H. Ding, Y. Gao, D. Natelson, Phys. Rev. B 2005, 72, 235302.

[12] N. Koch, S. Duhm, J. P. Rabe, A. Vollmer, R. L. Johnson, Phys. Rev. Lett. 2005, 95, 237601.

[13] B. H. Hamadani, D. A. Corley, J. W. Ciszek, J. M. Tour, D. Natelson, Nano Lett. 2006, 6, 1303.

[14] P. K. H. Ho, J. S. Kim, J. H. Burroughes, H. Becker, S. F. Y. Li, T. M. Brown, F. Cacialli, R. H. Friend, Nature 2000, 404, 481.

[15] L. S. Hung, C. W. Tang, M. G. Mason, Appl. Phys. Lett. 1997, 70, 152. 


\section{WILEY-VCH}

[16] Y. H. Zhou, C. Fuentes-Hernandez, J. Shim, J. Meyer, A. J. Giordano, H. Li, P.

Winget, T. Papadopoulos, H. Cheun, J. Kim, M. Fenoll, A. Dindar, W. Haske, E. Najafabadi,

T. M. Khan, H. Sojoudi, S. Barlow, S. Graham, J. L. Bredas, S. R. Marder, A. Kahn, B.

Kippelen, Science 2012, 336, 327.

[17] Y. L. Shen, M. W. Klein, D. B. Jacobs, J. C. Scott, G. G. Malliaras, Phys. Rev. Lett. 2001, 86, 3867.

[18] U. N. Maiti, W. J. Lee, J. M. Lee, Y. Oh, J. Y. Kim, J. E. Kim, J. Shim, T. H. Han, S.

O. Kim, Adv. Mater. 2014, 26, 40.

[19] L. Lindell, A. Burquel, F. L. E. Jakobsson, V. Lemaur, M. Berggren, R. Lazzaroni, J.

Cornil, W. R. Salaneck, X. Crispin, Chem. Mater. 2006, 18, 4246.

[20] G. Dennler, M. C. Scharber, C. J. Brabec, Adv. Mater. 2009, 21, 1323.

[21] H. Yan, Z. Chen, Y. Zheng, C. Newman, J. R. Quinn, F. Dötz, M. Kastler, A. Facchetti, Nature 2009, 457, 679.

[22] P. H. Wobkenberg, D. D. C. Bradley, D. Kronholm, J. C. Hummelen, D. M. de Leeuw, M. Colle, T. D. Anthopoulos, Synth. Metals 2008, 158, 468; S. Fabiano, C. Musumeci, Z. H. Chen, A. Scandurra, H. Wang, Y. L. Loo, A. Facchetti, B. Pignataro, Adv. Mater. 2012, 24, 951.

[23] G. Li, R. Zhu, Y. Yang, Nature Photon. 2012, 6, 153; S. Fabiano, Z. Chen, S. Vahedi, A. Facchetti, B. Pignataro, M. A. Loi, J. Mater. Chem. 2011, 21, 5891.

[24] L. A. A. Pettersson, S. Ghosh, O. Inganas, Org. Electron. 2002, 3, 143.

[25] K. Jeuris, L. Groenendaal, H. Verheyen, F. Louwet, F. C. De Schryver, Synth. Metals 2003, 132, 289.

[26] Y. Xuan, M. Sandberg, M. Berggren, X. Crispin, Org. Electron. 2012, 13, 632.

[27] CRC Handbook of Chemistry and Physics, 94th ed. 2013-2014, http://www.hbcpnetbase.com. 


\section{WILEY-VCH}

[28] G. Beamson, D. Briggs, High Resolution XPS of Organic Polymers: The Scienta ESCA300 Satabase, John Wiley \& Sons Ltd.: New York, 1992.

[29] G. Zotti, S. Zecchin, G. Schiavon, F. Louwet, L. Groenendaal, X. Crispin, W.

Osikowicz, W. Salaneck, M. Fahlman, Macromolecules 2003, 36, 3337; X. Crispin, S.

Marciniak, W. Osikowicz, G. Zotti, A. W. D. Van der Gon, F. Louwet, M. Fahlman, L.

Groenendaal, F. De Schryver, W. R. Salaneck, J. Polym. Sci. Part B-Polym. Phys. 2003, 41, 2561.

[30] T. Johansson, L. A. A. Pettersson, O. Inganas, Synth. Metals 2002, 129, 269.

[31] P. Wei, J. H. Oh, G. F. Dong, Z. N. Bao, J. Am. Chem. Soc. 2010, 132, 8852; N. Cho,

H. L. Yip, J. A. Davies, P. D. Kazarinoff, D. F. Zeigler, M. M. Durban, Y. Segawa, K. M.

O'Malley, C. K. Luscombe, A. K. Y. Jen, Adv. Energy Mater. 2011, 1, 1148.

[32] Y. B. Qi, S. K. Mohapatra, S. B. Kim, S. Barlow, S. R. Marder, A. Kahn, Appl. Phys.

Lett. 2012, 100, 4.

[33] S. Tanuma, C. J. Powell, D. R. Penn, Surf. Interface Anal. 2011, 43, 689.

[34] Z. He, C. Zhong, X. Huang, W. Y. Wong, H. Wu, L. Chen, S. Su, Y. Cao, Adv. Mater. 2011, 23, 4636.

[35] C. Duan, W. Cai, B. B. Y. Hsu, C. Zhong, K. Zhang, C. Liu, Z. Hu, F. Huang, G. C. Bazan, A. J. Heeger, Y. Cao, Energy Environ. Sci. 2013, 6, 3022.

[36] http://webbook.nist.gov/chemistry/.

[37] P. Gerbaux, L. Lamote, Y. V. Haverbeke, R. Flammang, J. M. Brown, Eur. J. Mass Spectrom. 2012, 18, 93. 
A

C<smiles>CC(N)CN(CCN)CCNCCN(CCNCCN)CCN(CCN(CCN)CCN)CCN(CCN)CCN</smiles>

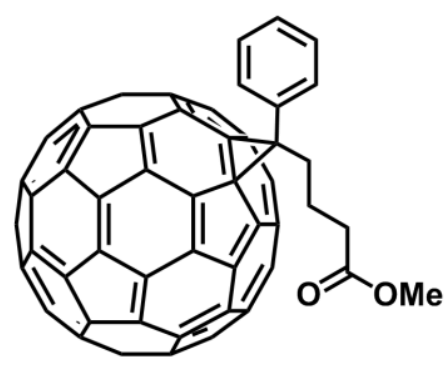

B

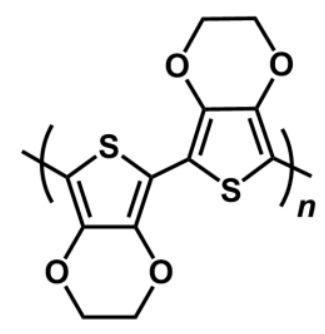<smiles>CC(C)(C)C1CC(C(C)(C)C)C2c3ccc(S(=O)(=O)O)cc3C(C(C)(C)C)c3c(S(=O)(=O)O)ccc1c32</smiles>

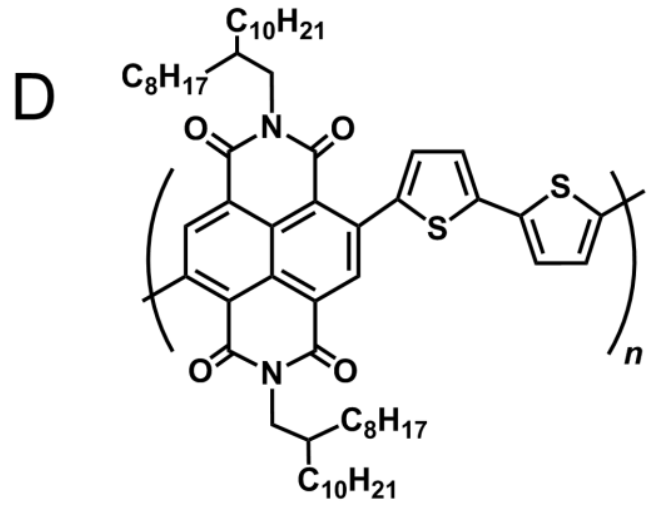

Scheme 1. Chemical structure of (a) highly branched PEI, (b) PEDOT-PSS, (c) PCBM, and (d) P(NDI2OD-T2). 
A

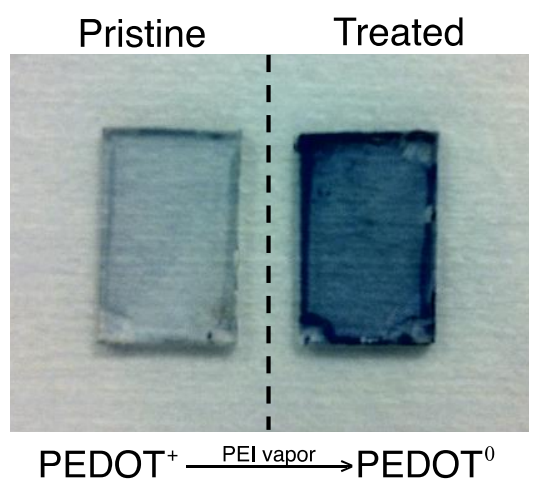

B

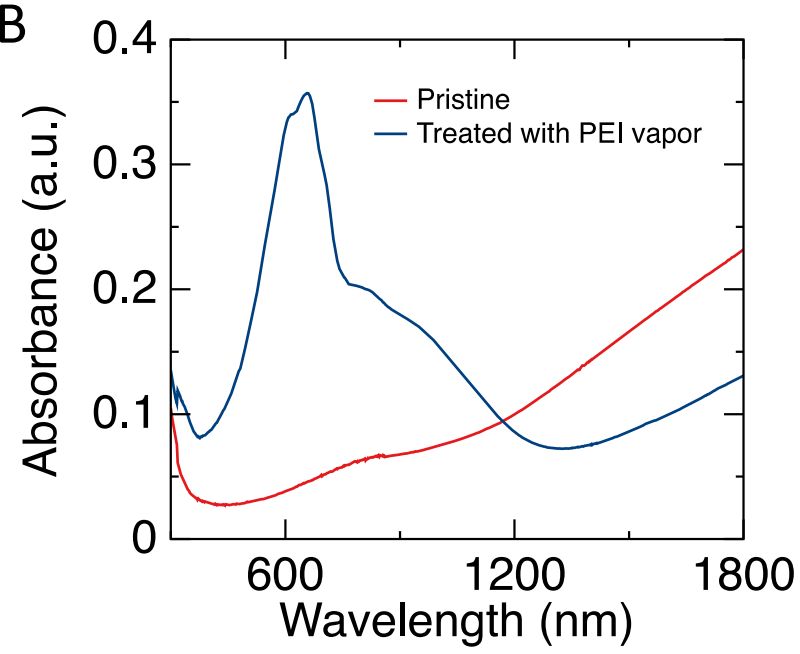

Figure 1. (a) Photographic image of pristine and PEI vapor-treated PEDOT-PSS films on glass substrates. (b) Absorption spectra of PEDOT-PSS films before (red line) and after (blue line) PEI vapor treatment. 

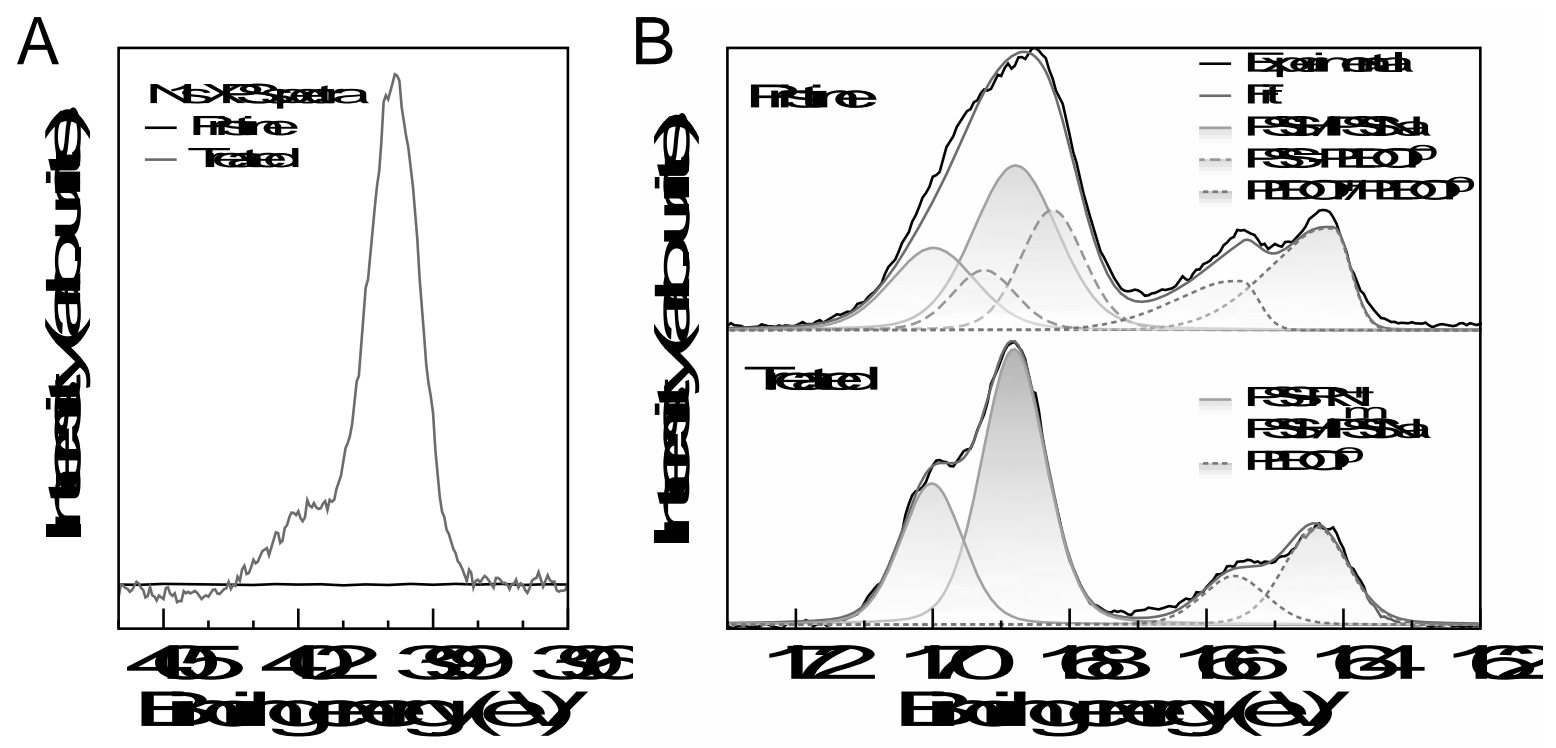

Figure 2. (a) N1 $s$ XPS spectra of pristine and PEI-vapor treated PEDOT-PSS films. (b) S2p XPS spectra of pristine and PEI-vapor treated PEDOT-PSS films. The two peaks at high binding energy $(171-167 \mathrm{eV})$ are from PSS, whereas the doublet $(167-163 \mathrm{eV})$ is from PEDOT. 

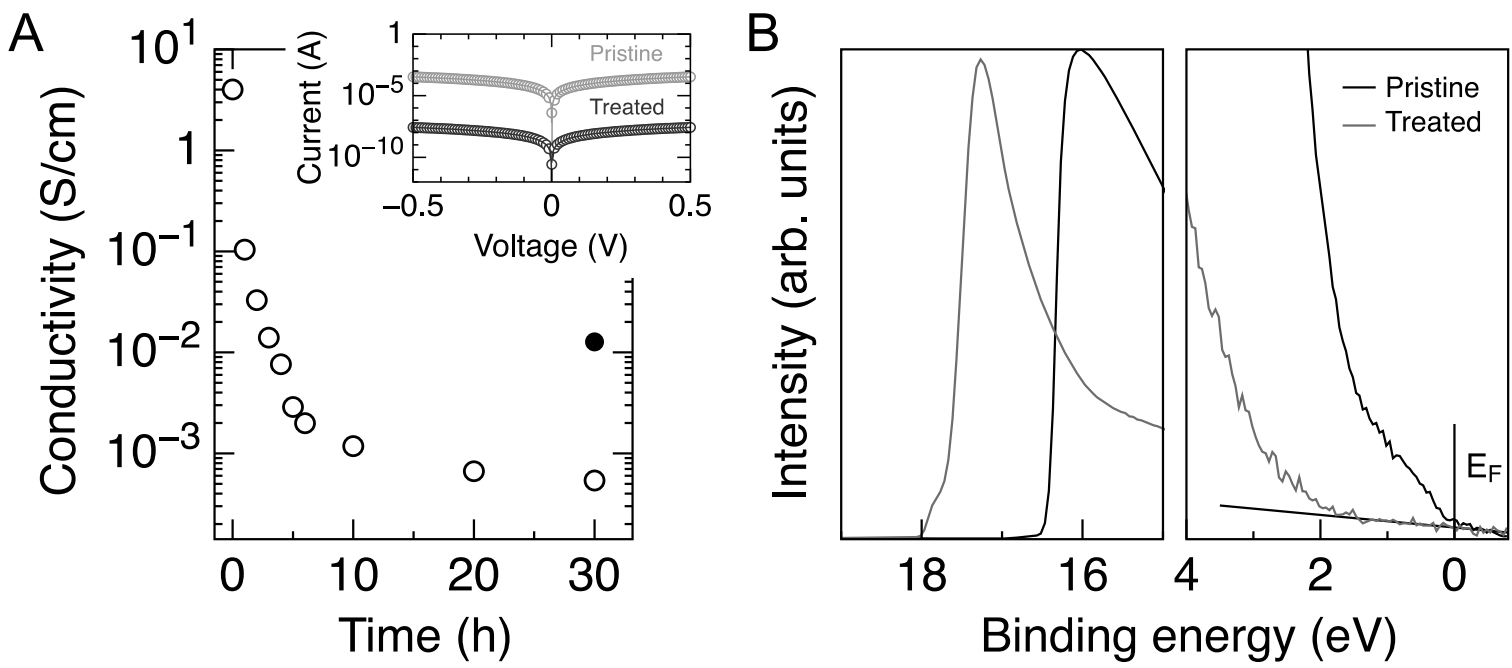

Figure 3. (a) Time-dependent variation of the electrical conductivity during exposure to the PEI vapor for PEDOT-PSS films. The filled circle represents the conductivity recovery after retesting the sample in air. The insets show the current-voltage characteristics for the PEDOTPSS film measured in a two-probe geometry. The solid line is a linear fit to the experimental data (see Figure S4 for the lin-lin plot). (b) Secondary electron cutoff (SECO, left) and UPS valence band spectra (HeI radiation, right) of pristine and PEI-vapor treated PEDOT-PSS films. 

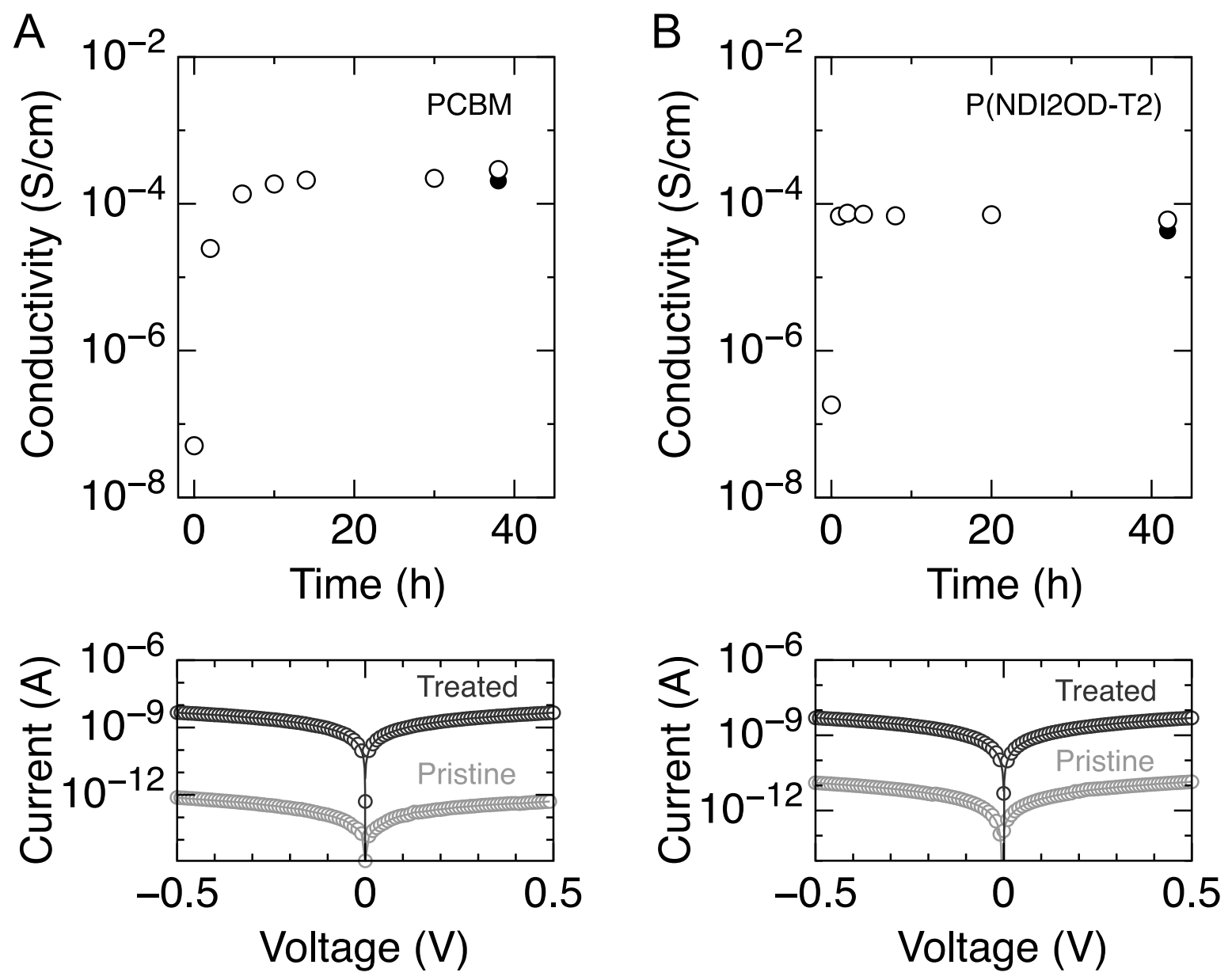

Figure 4. Time-dependent variation of the four-point-probe electrical conductivity during exposure to PEI vapor (top) and current-voltage characteristics measured in a two-probe geometry (bottom) for PCBM (a) and P(NDI2OD-T2) (b) films. The filled circle represents conductivity recovery test after storing the sample in vacuum $\left(\sim 10^{-7} \mathrm{mbar}\right)$. The solid line in the current-voltage characteristics is a linear fit to the experimental data (see Figure S4 for the lin-lin plot). 


\section{WILEY-VCH}
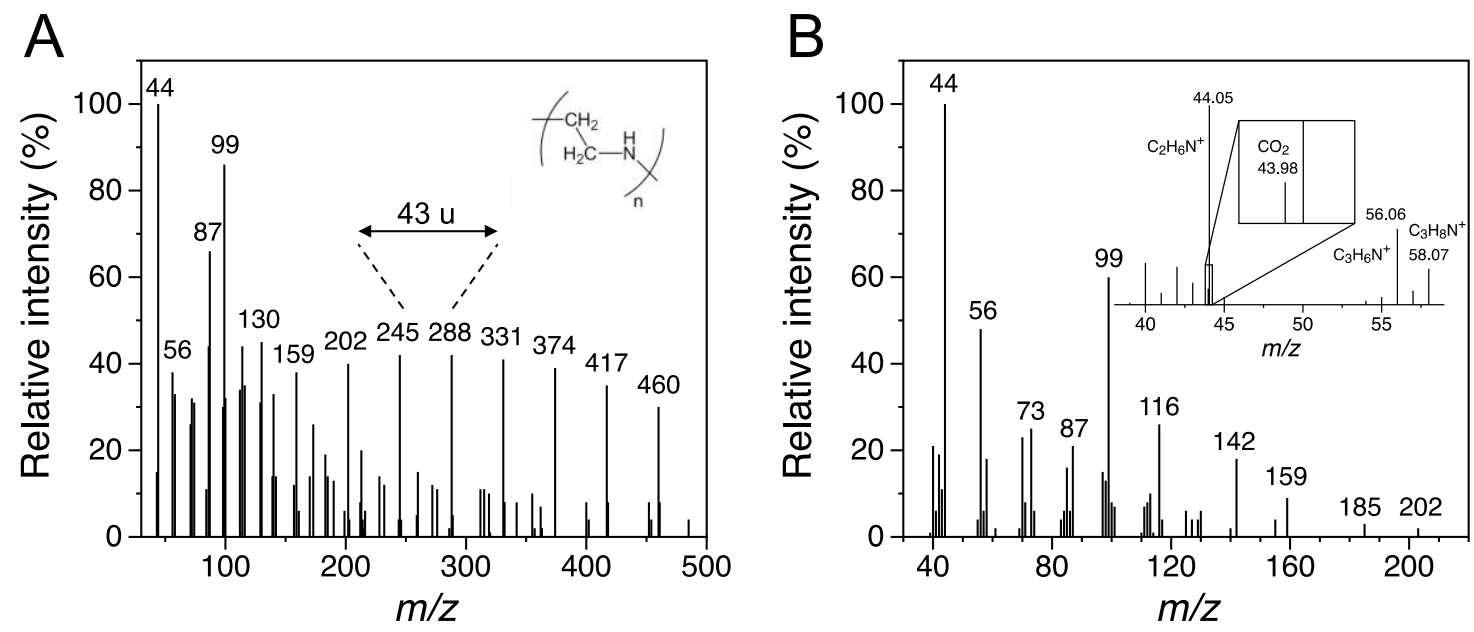

Figure 5. Direct Introduction Electron Ionization Mass Spectrometry (DI-EI-MS) analysis of the low mass $\left(a, M_{n} 600\right)$ and high mass (b, $M_{n}$ 10.000) PEI samples: full scan mass spectrum of the volatile molecules above the PEI condensed phase (sampling pressure $10^{-5}$ mbar). 


\section{WILEY-VCH}

Volatile impurities contained in polyethyleneimine (PEI), and identified as ethyleneimine dimers and trimers, are reported. These N-based molecules show a strong reducing character, as demonstrated by the change in electrical conductivity of organic (semi)conductors exposed to the PEI vapor. The results prove that electron transfer rather than dipole effect at the electrode interface is the origin of work function modification by PEI-based layers.

\section{Keyword: Doping}

Simone Fabiano, Slawomir Braun, Xianjie Liu, Eric Weverberghs, Pascal Gerbaux, Mats Fahlman, Magnus Berggren and Xavier Crispin*

Poly(ethylene imine) impurities induce n-doping reaction in organic (semi)conductors

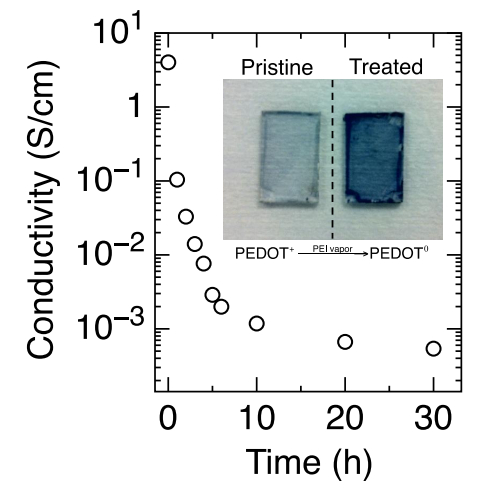




\section{WILEY-VCH}

Copyright WILEY-VCH Verlag GmbH \& Co. KGaA, 69469 Weinheim, Germany, 2013.

\section{Supporting Information}

Poly(ethylene imine) impurities induce n-doping reaction in organic (semi)conductors

Simone Fabiano, Slawomir Braun, Xianjie Liu, Eric Weverberghs, Pascal Gerbaux, Mats Fahlman, Magnus Berggren and Xavier Crispin*

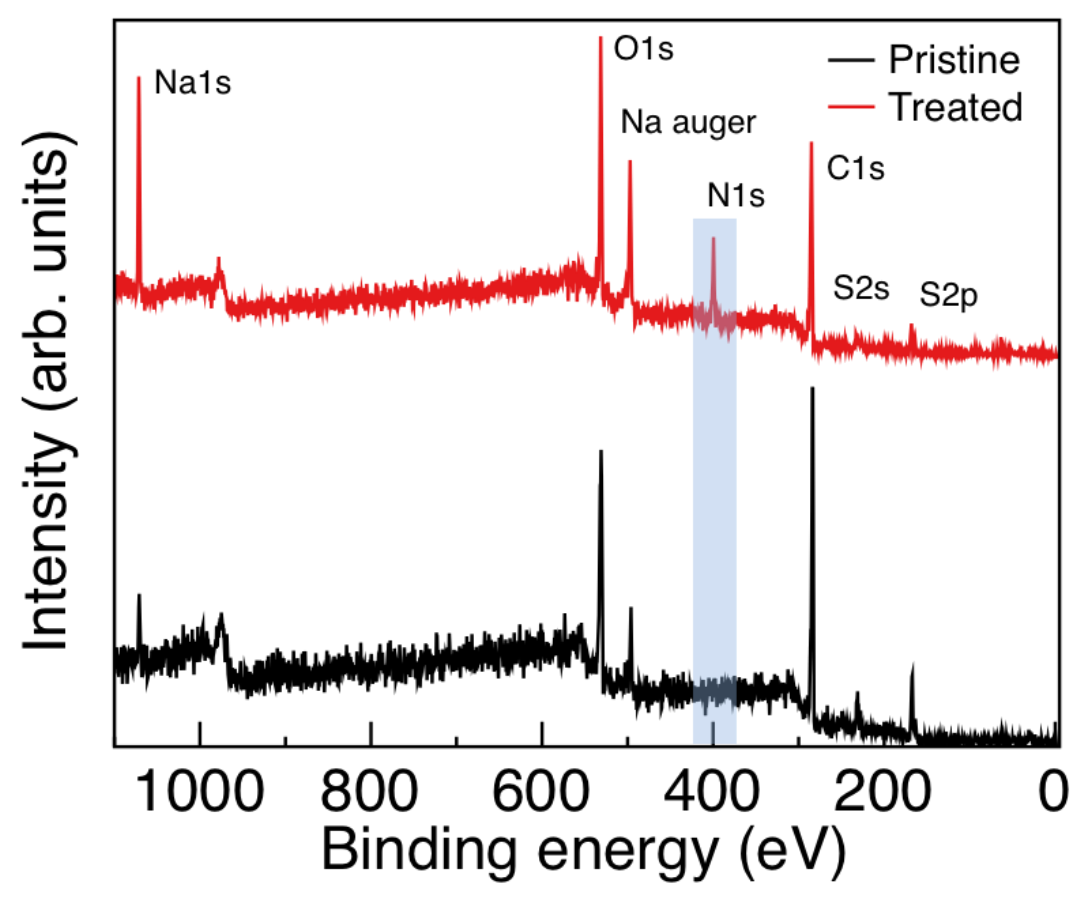

Figure S1. XPS survey spectra of pristine and PEI-vapor treated PEDOT-PSS films normalized to the $\mathrm{C} 1 s$ peak. 


\section{WILEY-VCH}
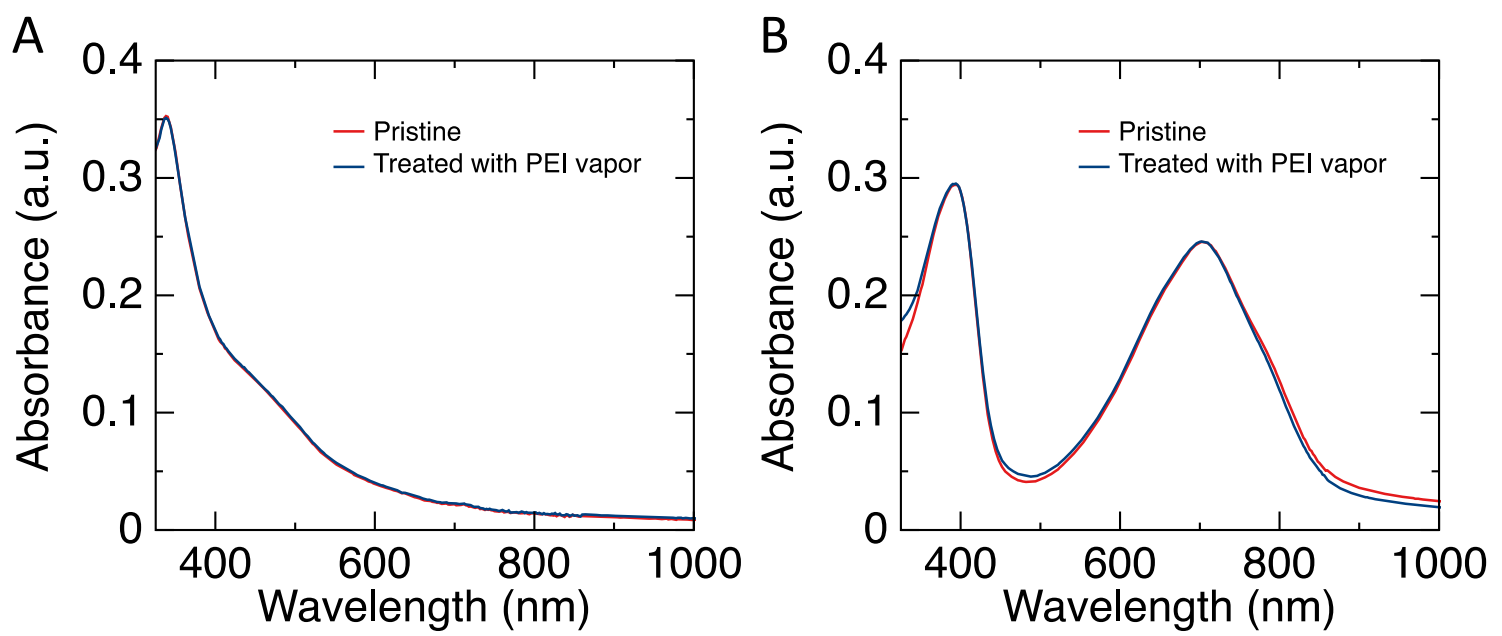

Figure S2. Absorption spectra of PCBM (a) and P(NDI2OD-T2) (b) films before (red line) and after (blue line) PEI vapor treatment. 

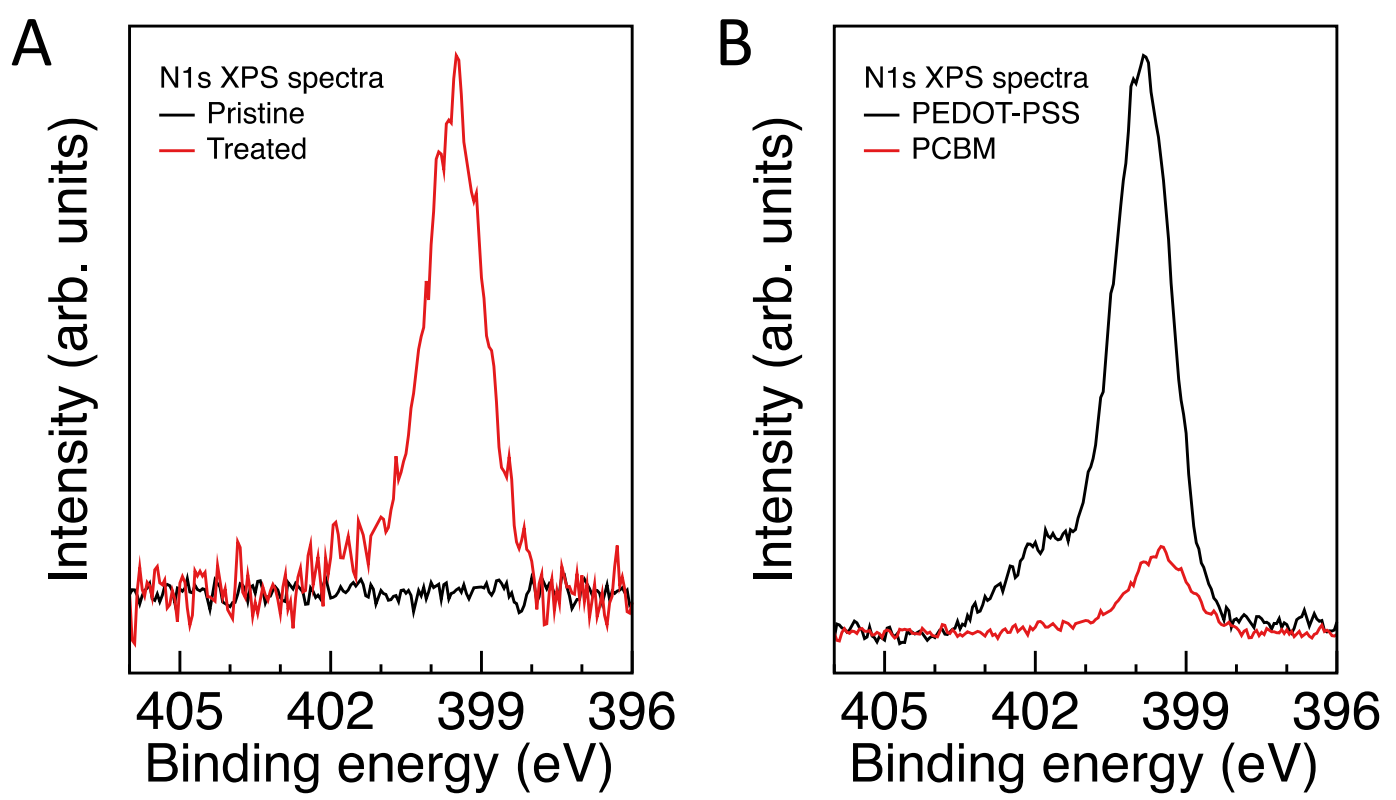

Figure S3. (a) N1s XPS spectra of pristine and PEI-vapor treated PCBM films. (b) Comparison between N1s XPS spectra of PEI-vapor treated PCBM and PEDOT-PSS films. 

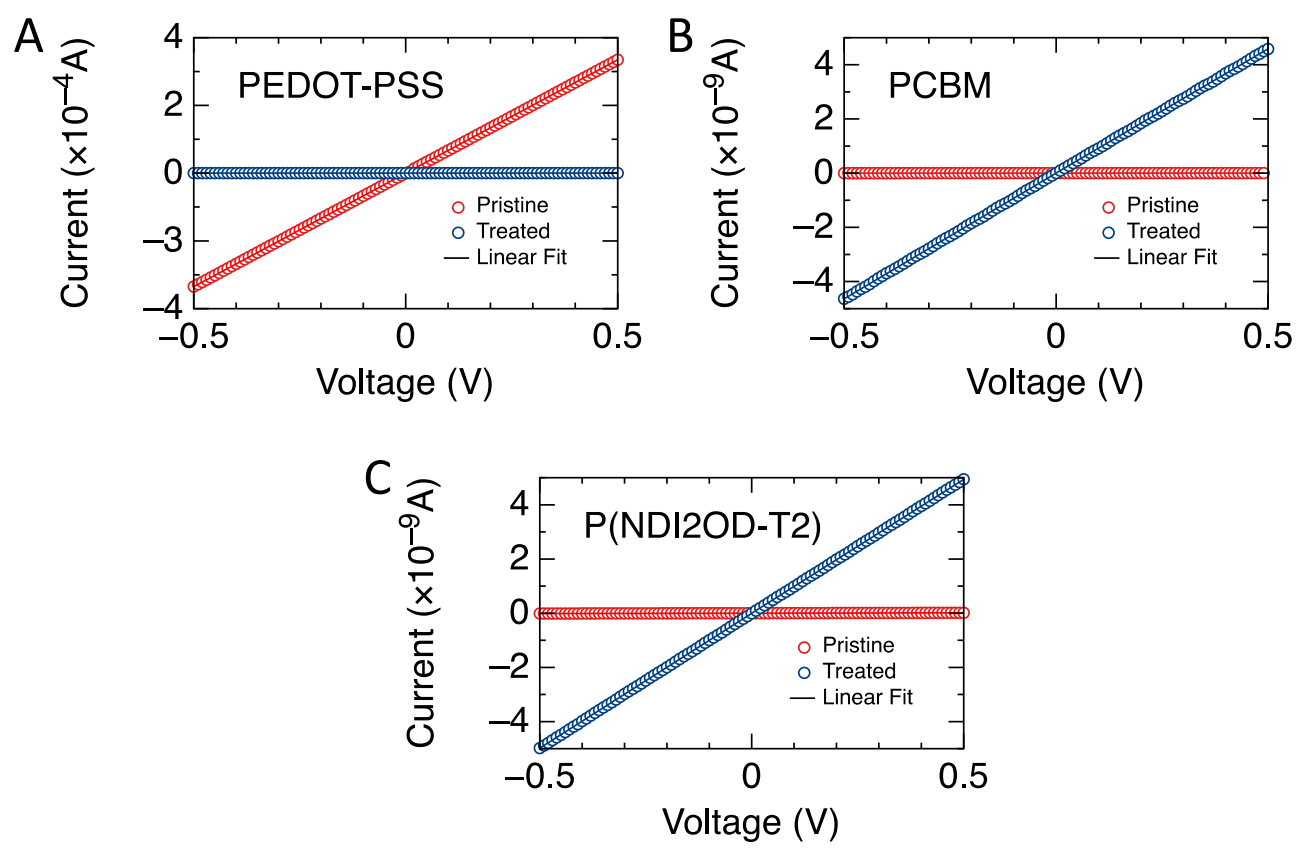

Figure S4. Lin-lin plot of the two-probe current-voltage characteristics presented in Fig. 3a (a), Fig. 4a (b) and Fig. 4b (c). 


\section{WILEY-VCH}

Table S1. DI-EI-MS composition analysis of the PEI samples reported in Figure 5.

\begin{tabular}{l|l|l|l|}
\hline $\mathrm{m} / \mathrm{x}$ & composition & $\mathrm{m} / \mathrm{x}$ & composition \\
\hline 44,05 & $\mathrm{C}_{2} \mathrm{H}_{6} \mathrm{~N}^{+}$ & 99,09 & $\mathrm{C}_{5} \mathrm{H}_{21} \mathrm{~N}_{2}^{+}$ \\
56,07 & $\mathrm{C}_{3} \mathrm{H}_{6} \mathrm{~N}^{+}$ & 116,12 & $\mathrm{C}_{5} \mathrm{H}_{12} \mathrm{~N}_{3}^{+}$ \\
73,07 & $\mathrm{C}_{3} \mathrm{H}_{8} \mathrm{~N}_{2}^{+}$ & 142,13 & $\mathrm{C}_{7} \mathrm{H}_{20} \mathrm{~N}_{4}^{+}$ \\
& & & $\mathrm{C}_{6} \mathrm{H}_{17} \mathrm{~N}_{5}^{+}$ \\
\hline 87,09 & $\mathrm{C}_{4} \mathrm{H}_{12} \mathrm{~N}_{2}^{+}$ & & \\
\hline
\end{tabular}




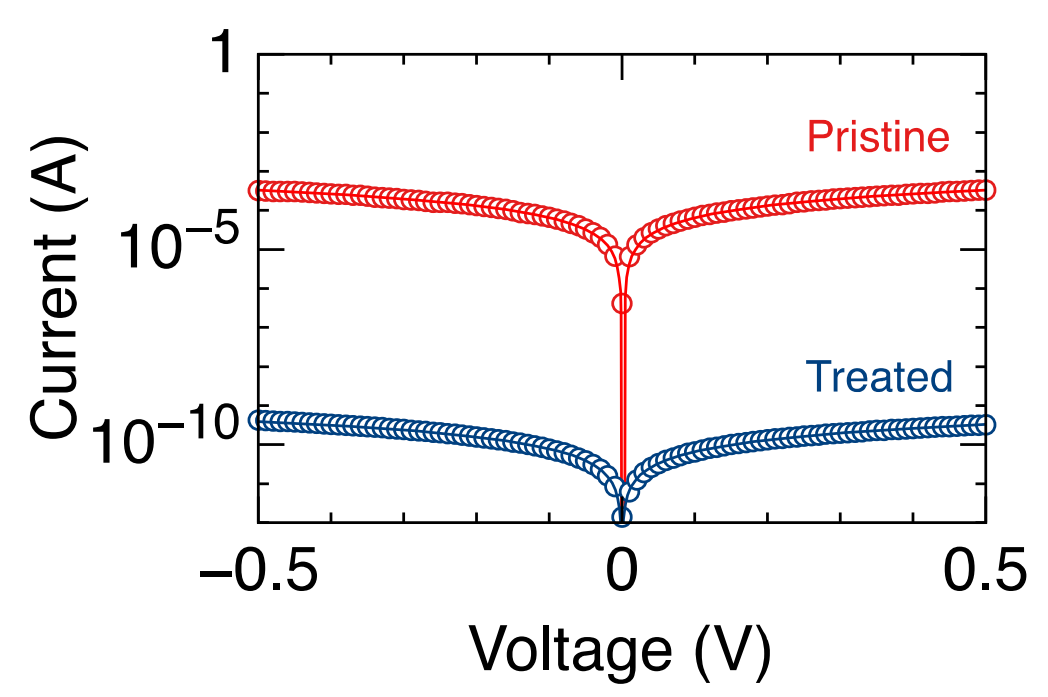

Figure S5. Current-voltage characteristics measured in a two-probe geometry for PEDOT-PSS films after $1 \mathrm{~h}$ exposure to pure diethylenetriamine vapor. The solid line is a linear fit to the experimental data. 